\title{
Frontal lobe syndrome reassessed: comparison of patients with lateral or medial frontal brain damage
}

\author{
Sergio Paradiso, Eran Chemerinski, Kazim M Yazici, Armando Tartaro,
} Robert G Robinson

\begin{abstract}
Examination of mood and behaviour changes after frontal damage may contribute to understanding the functional role of distinct prefrontal areas in depression and anxiety. Depression and anxiety disorders, symptoms, and behaviour were compared in eight patients with single lateral and eight patients with single medial frontal lesions matched for age, sex, race, education, socioeconomic status, side, and aetiology of lesion 2 weeks and 3 months after brain injury. DSM IV major depressive and generalised anxiety disorders were more frequent in patients with lateral compared with medial lesions at 2 weeks but not at 3 months. At 3 months, however, patients with lateral damage showed greater severity of depressive symptoms, and greater impairment in both activities of daily living and social functioning. At initial evaluation depressed mood and slowness were more frequent, whereas at 3 months slowness, lack of energy, and social unease were more frequent in the lateral than the medial group. Patients with lateral lesions showed greater reduction of emotion and motivation (apathy) during both examinations. Medial frontal injury may fail to produce emotional dysregulation or may inhibit experience of mood changes, anxiety, or apathy. Lateral prefrontal damage may disrupt mood regulation and drive while leaving intact the ability to experience (negative) emotions.

(F Neurol Neurosurg Psychiatry 1999;67:664-667)
\end{abstract}

Keywords: frontal lobe; depression; anxiety; apathy; disinhibition

Prefrontal dysfunction has been found in mood disorders and anxiety. Neuroimaging, however, has failed to consistently identify specific locations and types of functional changes within the prefrontal cortex associated with depression $^{1-5}$ or anxiety. ${ }^{7}$

The prefrontal lobe has been divided into dorsal lateral, medial, and orbital cortices. ${ }^{8}$ Patients with lateral prefrontal damage may show apathy, indifference, speech poverty, and poor executive abilities, ${ }^{4}$ and with acute left hemispheric injuries, major depression. ${ }^{9}$ Damage to the orbital prefrontal cortex may lead to poor impulse control, puerility, euphoria, increased energy, aggression, and violence. ${ }^{10}$ The orbital cortex can be divided into medial and lateral subregions. The medial subregion connects with limbic areas whereas the lateral subregion connects with the primary and sensory association cortex. ${ }^{11}$ For its limbic connections, ${ }^{11}$ the medial prefrontal wall resembles the medial subregion of the orbital cortex. Large lesions of the frontal medial walls (and anterior cingulate) have been associated with apathy ${ }^{12}$ but emotional and instinctual disorders as well as sociopathy have been associated with damage to the confluence of the medial and orbital cortex. ${ }^{13}$

Much of our knowledge of the psychiatric consequences of lesions involving the medial prefrontal cortex in humans is based on case report studies. ${ }^{8}$ This literature tends to select positive findings. We therefore report on the first systematic examination of depression, anxiety, apathy, disinhibition, and psychosocial functioning at 2-4 weeks and at 3 months after injury in patients with lateral or medial damage to the prefrontal cortex. We hypothesised that medial frontal injury would lead to euphoria and agitation, whereas lateral injuries would lead to depression. No selective association of either medial or lateral lesions with apathy was predicted. $^{8}{ }^{12}$

\section{Methods}

Data from about 360 CT scans were reviewed by a neurologist, a neuroradiologist (AT), and a psychiatrist with experience in neuroanatomy (KY) blind to the other measures. Scans were from a consecutive series of prospectively evaluated patients admitted to the Shock Trauma Center of the Maryland Institute of Emergency Medical Services System (MIEMSS) for traumatic brain injury, or the University of Maryland Hospital for acute stroke, or the Younker's Rehabilitation Hospital in Des Moines, Iowa following acute stroke.

The inclusion criterion was a single lateral or medial (including anterior cingulate gyrus) 

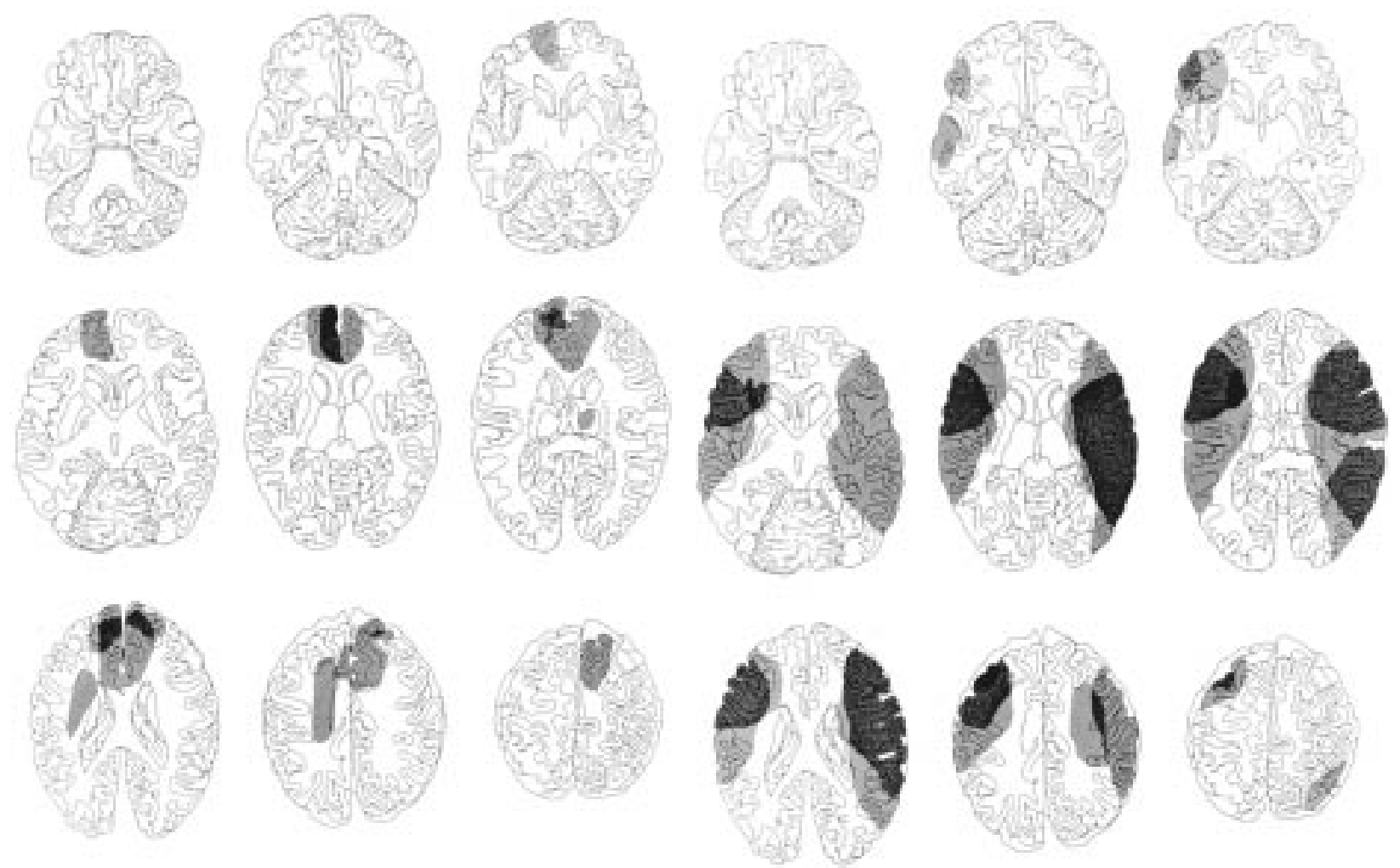

Schematic templates of CT slices showing cross sectional areas of lesion for patients with medial (left) and lateral (right) frontal lesions. Darker areas indicate greater across subject lesion overlap.

frontal lesion as ascertained by all raters. Scans were performed using GE 9900, AS and E 500, and EMI 1010 scanners (stroke patients from Baltimore), GE 1010 (trauma patients from Baltimore), and Picker international 1200 or Somaton plus $24 \mathrm{~B} 30 \mathrm{~B}$ (stroke patients from Iowa) without contrast at 0 degrees angle from the canthomeatal line. Assessment of lesion location involved transposing the area of ischaemia as visualised on CT onto standard templates of axial brain slices (figure). ${ }^{14}$

Exclusion criteria were: history of other neurological disorders or life threatening physical illness, CT evidence of previous brain injury, decreased level of consciousness or medical instability at the time of interview, aphasic disorders (not able to follow a three stage command or complete the first part of the token test), penetrating head injury, associated spinal cord injury, multiple non-CNS involvement, or secondary brain damage due to severe hypotension or hypoxia.

Thirty two patients with lateral and 14 patients with medial lesions met these criteria. Eight of fourteen patients with medial frontal lesions were matched as closely as possible for age ( \pm 5 years), sex, education ( \pm 2 years), race, socioeconomic status ( \pm 1 social class), side, and aetiology of lesion with eight patients with lateral lesions. The two groups constituted the study sample. Six medial patients could not be matched or had incomplete data. A patient (medical group) had a small thalamic lesion.

PSYCHIATRIC EXAMINATION

After giving written informed consent, patients were administered quantitative psychiatric and psychosocial measures including the Hamilton rating scale for depression (Ham-D), the mini mental state examination (MMSE), and the Johns Hopkins functioning inventory (JHFI) measuring physical independence in activities of daily living (ADL, higher scores=greater impairment). Social functioning was assessed using the social ties checklist (STC) and the social functioning examination (SFE). The STC quantifies the number of social connections available to the patient (higher scores=less social support). The SFE assesses patients' satisfaction with their social functioning (higher scores=less satisfaction). A modified present state examination (PSE), ${ }^{15}$ was administered by a psychiatrist. A diagnosis of major or minor depression, or generalised anxiety disorder (GAD) was made by using DSM-IV criteria.

Seventeen PSE symptom clusters were calculated using the method described by Wing et $a l .{ }^{15}$ The presence and severity of disinhibition (agitation/irritability/violence, embarrassing behaviour/loss of social restraint, irreverent/ histrionic behaviour, hypomanic affect, pressured/non-social speech), and reduction in emotion and motivation (self-neglect, slowness/underactivity, mannerism/posturing, stereotypies/ catatonic movements, blunted affect, slow/restricted quantity of speech/ muteness) were assessed using the PSE.

STATISTICAL ANALYSIS

Data were analysed using means (SD), $F$ tests, and likelihood ratio (LR) $\chi^{2}$ for frequency distributions. Values of $\mathrm{p}$ are two tailed unless 
Severity of psychiatric symptoms, cognitive impairment, activities of daily living, and social functioning

\begin{tabular}{lllll}
\hline & Medial & Lateral & Effect size & Power \\
\hline Initial evaluation: & & & & \\
Ham D & $8.1(8.1)$ & $14.0(5.3)^{\star}$ & 0.86 & 0.49 \\
PSE & $10.5(13.2)$ & $24.1(17.6)^{\star \star}$ & 0.87 & 0.37 \\
MSE & $25.9(3.7)$ & $21.1(10.0)$ & 0.63 & 0.22 \\
JHFI & $2.9(3.4)$ & $7.1(8.1)$ & 0.67 & 0.24 \\
STC & $3.0(1.6)$ & $4.0(2.1)$ & 0.53 & 0.17 \\
SFE & $0.1(0.05)$ & $0.3(0.2)^{\star \star \star}$ & 1.13 & 0.72 \\
months: & & & & \\
Ham D & $5.7(3.7)$ & $9.6(3.6) \dagger$ & 1.06 & 0.51 \\
PSE & $6.0(6.8)$ & $10.2(6.5)$ & 0.63 & 0.22 \\
MMSE & $23.8(5.8)$ & $23.9(8.9)$ & 0.01 & 0.05 \\
JHFI & $1.6(2.3)$ & $5.2(4.1) \dagger$ & 1.09 & 0.52 \\
STC & $2.4(1.0)$ & $4.0(2.0) \ddagger$ & 0.71 & 0.27 \\
SFE & $0.1(.10)$ & $0.24(.16) \ddagger$ & 1.07 & 0.51 \\
\hline
\end{tabular}

${ }^{\star} t(14)=1.72, \mathrm{p}=0.05$ one tailed; ${ }^{\star \star} t(14)=1.75, \mathrm{p}=0.10 ;{ }^{\star \star \star} t(14)=2.78, \mathrm{p}<0.015$; other comparisons $\mathrm{p}>0.10$.

$\dagger t(14)=2.1, \mathrm{p}<0.06 ; \ddagger t(14)=1.9, \mathrm{p}<0.09$; other comparisons $\mathrm{p}>0.1$.

otherwise specified. Power and effect sizes (ES) are reported.

\section{Results}

BACKGROUND CHARACTERISTICS

Each group was composed of three women and five men, five right and three left hemispheric lesions, and three head injuries and five strokes. Mean age of the medial group was 39.5 years (SD 17, range 27-74), and that of the lateral group was 44.1 (SD 17, range 20-61). Five white, two African-American, and one Hispanic patient were in the medial, and six white and two African-American patients were in the lateral group. Five patients in the medial and four in the lateral group were of socioeconomic (Hollingshead) class IV and V. Both groups had 11.8 (SD 3) mean years of education. No statistically significant differences were found.

\section{PSYCHIATRIC EVALUATION}

Depression or anxiety

At initial evaluation, depression was more frequent in lateral (six or $75 \%$, three major, and three minor depression) than medial lesion patients (two or $25 \%$, one major, and one minor depression) $\left\{\mathrm{LR} \chi^{2}(1)=4.2, \mathrm{p}<0.05\right.$, power $=0.99, \mathrm{ES}=1.1\}$. Five $(62.5 \%)$ patients had GAD in the lateral and one $(12.5 \%)$ in the medial group $\left\{\operatorname{LR} \chi^{2}(1)=4.5, \quad \mathrm{p}<0.04\right.$, power $=0.98, E S=1.0\}$. Patients with lateral damage showed less satisfaction with their social functioning (table). At 3 months, seven patients $(87.5 \%)$ in both groups no longer fulfilled criteria for depression and/or GAD. However, patients with lateral damage showed greater severity of depressive symptoms, impairment in ADL, and psychosocial functioning (table). Comparing the symptom clusters from the PSE at initial evaluation, the lateral group showed a greater frequency of simple depression $\left\{\mathrm{LR} \chi^{2}(1)=4.2, \mathrm{p}<0.05\right.$, power $=$ $0.99, \mathrm{ES}=1.1\}$ and slowness $\left\{\operatorname{LR} \chi^{2}(1)=4.5\right.$, $\mathrm{p}<0.04$, power $=0.98, \mathrm{ES}=1.0\}$. At 3 months, patients with lateral damage showed higher frequency of slowness $\left\{\operatorname{LR} \chi^{2}(1)=6.9, p<0.01\right.$, power $=0.97, \mathrm{ES}=0.98\}$, and greater lack of energy and social unease both $\operatorname{LR} \chi^{2}(1)=4.8$, $\mathrm{p}<0.03$, power $=0.88, \mathrm{ES}=0.79\}$.
Disinhibition or hypomania

During the initial evaluation, four patients in the lateral (three displayed agitation and hostile irritability; one histrionic and hypomanic behaviour) and two in the medial group (one hypomanic affect, another agitation) had symptoms of disinhibition/hypomania. Total number of symptoms was fourfold greater (nine) in the lateral than in the medial group (two). The mean number of symptoms was 1.12 (SD 1.35) in the lateral and 0.25 (SD $0.46)$ in the medial group $(t \quad(14)=1.27$, $\mathrm{p}=0.10$, power $=0.36, \mathrm{ES}=0.86$ ). At 3 months, three patients in the lateral group and one patient in the medial group showed disinhibition/hypomania (NS, power $=0.07$, $\mathrm{ES}=0.12$ ).

\section{Reduction in emotion and motivation}

Five patients $(62.5 \%)$ in the lateral and one $(12.5 \%)$ in the medial group showed decreased emotion and motivation $\left\{\operatorname{LR} \chi^{2}(1)=4.55\right.$, $\mathrm{p}<0.04$, power $=0.98, \mathrm{ES}=1.0\}$. These patients showed a combination of severe self neglect, slowness, blunted affect, and decreased speech. One patient was almost mute at both evaluations. No patient in either group showed mannerisms, posturing, or stereotypies. At 3 months, five patients in the lateral group showed decreased emotion and motivation compared with none in the medial group $\{\mathrm{LR}$ $\chi^{2}(1)=9.29, \mathrm{p}<0.003$, power $\left.=0.99, \mathrm{ES}=1.3\right\}$.

\section{Discussion}

During the inhospital evaluation, patients with single lesions of the lateral prefrontal cortex showed more frequent depression and anxiety disorders than patients with medial frontal lesions and a greater frequency of symptoms of depression and anxiety at 3 months postinjury. Patients with lateral lesions also showed greater reduction of emotion and motivation/apathy at both evaluations.

Before discussing these findings, the limitations of our methods should be acknowledged. Due to a relatively few patients some important differences may have been missed. Although laterality questions were not examined, our findings were unlikely to be influenced by laterality effects because of the side matched group design. The aetiology of the brain injury may have influenced some of our findings such as duration of depression (trauma has been associated with shorter duration of depression than stroke) or the role of non-brain injury factors in depression. Patients with lateral damage had larger lesions. This may have influenced the greater functional impairment found in the lateral group and perhaps greater depression. However, physical impairment in activities of daily living was not significantly correlated with depression (data available from the authors) and depression has not been correlated with lesion volume in the acute poststroke period. ${ }^{16}$ Some of the larger lateral lesions involved part of temporal lobes and this may have influenced our findings.

A most surprising finding was that medial frontal lesions did not lead to higher euphoria, disinhibition, loss of emotion, or apathy than 
lateral lesions. The same instruments used in this study, however, were sensitive to increased cheerfulness and hypomania associated with right frontal compared with right temporal lesions. ${ }^{17}$ Apathy has been associated with extensive damage to the cingulum and mesial frontal areas as well as other structures. ${ }^{12} 18$ Decreased blood flow in the right dorsal lateral frontal and left frontotemporal cortices was found in 40 patients with apathy after stroke to diverse brain areas compared with nonapathetic stroke controls. ${ }^{19}$

The human prefrontal cortex has been shown to be involved in the experience ${ }^{20}$ and appraisal of emotion ${ }^{2122}$ as well as in primary and secondary mood disorders. No consensus has been achieved, however, regarding the specific location of the prefrontal dysfunction in mood disorders. ${ }^{1-4} 2324$

Cummings found personality but not mood changes after damage to the frontal- cingulate circuit. $^{12}$ Medial and ventral medial frontal cortex participates in the response to emotion evoking stimuli ${ }^{13}$ and selective attention to subjective emotion. ${ }^{25}$ Therefore, medial frontal activity may be necessary to experience emotion including depressed mood, anxiety, or apathy. Medial frontal injury may either not lead to emotional changes or may inhibit the perception of mood changes, anxiety, or apathy. ${ }^{8}$ Unilateral damage to the lateral prefrontal cortex may disrupt mood regulation and drive while leaving intact the ability to experience (disturbed) emotions.

This study was presented in part at the 9th Annual Meeting of the American Neuropsychiatry Association (Honolulu, Hawail, 1-3 February 1998). The work has been supported by NIH grants MH53592 and MH52879.

1 Uytdenhoef P, Portelange P, Jacquy J, et al. Regional cerebral blood flow and lateralized hemispheric dysfunction in depression. Br F Psychiatry 1983;143:128-32.

2 Baxter LR Jr, Phelps ME, Mazziotta JC, et al. Cerebral metabolic rates for glucose in mood disorders. Studies with positron emission tomography and fluorodeoxyglucose F 18. Arch Gen Psychiatry 1985;42:441-7.

3 Martinot JL, Hardy P, Feline A, et al. Left prefrontal glucose hypometabolism in the depressed state: a confirmation. $A m$ f Psychiatry 1990;147:1313-7.
4 Dolan RJ, Bench CJ, Liddle PF, et al. Dorsolateral prefrontal cortex dysfunction in the major psychoses; symptom or disease specificity? f Neurol Neurosurg Psychiatry 1993;56: $1290-4$

5 Drevets WC, Videen TO, Price JL, et al. A functional anatomical study of unipolar depression. $\mathcal{f}$ Neurosci 1992;12:3628-41.

6 Reiman EM. The application of positron emission tomography to the study of normal and pathologic emotions. 7 Clin Psychiatry 1997;16:4-12.

7 Fredrikson M, Wik G, Annas P, et al. Functional neuroanatomy of visually elicited simple phobic fear: additional data and theoretical analysis. Psychophysiology 1995; 32:43-8.

8 Fuster JM. The prefrontal cortex. Anatomy, physiology, and neuropsychology of the frontal lobe. 3rd ed. Philadelphia: Lippincot-Raven, 1997.

9 Robinson R, Kubos K, Starr L, et al. Mood disorders in stroke patients: importance of lesion location. Brain 1984; 107:81-93.

10 Grafman J, Schwab K, Warden D, et al. Frontal lobe injuries, violence, and aggression: a report of the Vietnam head injury study. Neurology 1996;46:1231-8.

11 Carmichael ST, Price JL. Limbic connections of the orbital and medial prefrontal cortex in macaque monkeys. f Comp Neurol 1995;363:615-41.

12 Cummings JL. Frontal-subcortical circuits and human behavior. Arch Neurol 1993;50:873-80.

13 Damasio AR. On some functions of the human prefrontal cortex. In: Grafman J, Holyoak KJ, Boller F, eds. Structure and functions of the human prefrontal cortex. New York: Ann N Y Acad Sci 1995;769:241-51.

14 Levine DN, Grek A. The anatomic basis of delusion after right cerebral infarction. Neurology 1984;34:577-82.

15 Wing J, Cooper E, Sartorius N, eds. Measurement and classification of psychiatric symptoms. Cambridge: Cambridge University Press, 1974 .

16 Dam H, Pedersen HE, Ahigren P. Depression among patients with stroke. Acta Psychiatr Scand 1989;80:118-24.

17 Starkstein SE, Robinson RG, Honig MA, et al. Mood changes after right-hemisphere lesions. $\mathrm{Br} \mathcal{F}$ Psychiatry 1989;155:79-85

18 Marin RS. Apathy: a neuropsychiatric syndrome. 7 Neuropsychiatry Clin Neurosci 1991;3:243-54.

19 Okada K, Kobayashi S, Yamagata S, et al. Post-stroke apathy and regional cerebral blood flow. Stroke 1997;28:2437-41.

20 George MS, Ketter TA, Parekh PI, et al. Brain activity during transient sadness and happiness in healthy women. $\mathrm{Am}$ 7 Psychiatry 1995;152:341-51.

21 Paradiso S, Robinson RG, Andreasen NC, et al. Emotional activation of limbic circuitry in elderly normal subjects in a PET study. Am F Psychiatry 1997;154:384-9.

22 Paradiso S, Johnson DL, Andreasen NC, et al. Cerebral blood flow changes associated with attribution of emotional valence to pleasant, unpleasant, and neutral visual stimuli. Am F Psychiatry 1999 (in press).

23 Drevets WC, Price JL, Simpson JR, Jr, et al. Subgenual prefrontal cortex abnormalities in mood disorders. Nature 1997;386:824-7.

24 Mayberg HS, Lewis PJ, Regenold W, et al. Paralimbic hypoperfusion in unipolar depression. $\mathcal{F} \mathrm{Nucl} \mathrm{Med} \mathrm{1994;35:}$ 929-34.

25 Lane RD, Fink GR, Chau P M-L, et al. Neural activation during selective attention to emotional responses. Neuroreport 1997;8:3969-72. 